\title{
MULTIPLICITY RESULTS FOR ASYMMETRIC BOUNDARY VALUE PROBLEMS WITH INDEFINITE WEIGHTS
}

\author{
FRANCESCA DALBONO
}

Received 21 October 2003

We prove existence and multiplicity of solutions, with prescribed nodal properties, to a boundary value problem of the form $u^{\prime \prime}+f(t, u)=0, u(0)=u(T)=0$. The nonlinearity is supposed to satisfy asymmetric, asymptotically linear assumptions involving indefinite weights. We first study some auxiliary half-linear, two-weighted problems for which an eigenvalue theory holds. Multiplicity is ensured by assumptions expressed in terms of weighted eigenvalues. The proof is developed in the framework of topological methods and is based on some relations between rotation numbers and weighted eigenvalues.

\section{Introduction and statement of the main result}

This paper is devoted to the study of the existence and multiplicity of solutions to an asymmetric, "asymptotically linear" two-point boundary value problem of the form

$$
\begin{gathered}
u^{\prime \prime}(t)+f(t, u(t))=0 \\
u(0)=0=u(T)
\end{gathered}
$$

where $T>0$ is fixed and $f:[0, T] \times \mathbb{R} \rightarrow \mathbb{R}$ satisfies the Carathéodory conditions.

By a solution to (1.1) we mean an absolutely continuous function $u:[0, T] \rightarrow \mathbb{R}$ satisfying (1.1) and such that its derivative $u^{\prime}$ is absolutely continuous.

Before describing the asymptotically linear assumptions, we need to introduce some notation. We first define $g^{+}(t):=\max \{g(t), 0\}$ for any given function $g \in \mathrm{L}^{1}([0, T], \mathbb{R})$. Moreover, for every $i \in\{1,2\}$ and for every $\varphi_{i}, \psi_{i} \in \mathrm{L}^{1}([0, T], \mathbb{R})$, by $\left(\varphi_{1}, \psi_{1}\right) \geq\left(\varphi_{2}, \psi_{2}\right)$ we mean that $\varphi_{1}(t) \geq \varphi_{2}(t)$ and $\psi_{1}(t) \geq \psi_{2}(t)$ for a.e. $t \in[0, T]$. According to the notation of [53], we write $\left(\varphi_{1}, \psi_{1}\right) \succ\left(\varphi_{2}, \psi_{2}\right)$ if $\left(\varphi_{1}, \psi_{1}\right) \geq\left(\varphi_{2}, \psi_{2}\right)$ and $\varphi_{1}(t)>\varphi_{2}(t), \psi_{1}(t)>\psi_{2}(t)$ on a common subset of $[0, T]$ of positive measure.

In what follows, we assume the following asymmetric conditions.

$\left(\mathrm{H}^{ \pm}\right)$There exist $a_{\infty}^{+}, a_{\infty}^{-}, b_{\infty}^{+}, b_{\infty}^{-} \in \mathrm{L}^{1}([0, T], \mathbb{R})$ with $\left(\left(a_{\infty}^{+}\right)^{+},\left(a_{\infty}^{-}\right)^{+}\right) \succ(0,0)$ on $(0, T)$ such that 


$$
\begin{aligned}
& a_{\infty}^{+}(t) \leq \liminf _{x \rightarrow+\infty} \frac{f(t, x)}{x} \leq \limsup _{x \rightarrow+\infty} \frac{f(t, x)}{x} \leq b_{\infty}^{+}(t), \\
& a_{\infty}^{-}(t) \leq \liminf _{x \rightarrow-\infty} \frac{f(t, x)}{x} \leq \limsup _{x \rightarrow-\infty} \frac{f(t, x)}{x} \leq b_{\infty}^{-}(t)
\end{aligned}
$$

uniformly a.e. in $t \in[0, T]$.

$\left(\mathrm{K}^{ \pm}\right)$There exist $a_{0}^{+}, a_{0}^{-}, b_{0}^{+}, b_{0}^{-} \in \mathrm{L}^{1}([0, T], \mathbb{R})$ with $\left(\left(a_{0}^{+}\right)^{+},\left(a_{0}^{-}\right)^{+}\right) \succ(0,0)$ on $(0, T)$ such that

$$
\begin{aligned}
& a_{0}^{+}(t) \leq \liminf _{x \rightarrow 0^{+}} \frac{f(t, x)}{x} \leq \limsup _{x \rightarrow 0^{+}} \frac{f(t, x)}{x} \leq b_{0}^{+}(t), \\
& a_{0}^{-}(t) \leq \liminf _{x \rightarrow 0^{-}} \frac{f(t, x)}{x} \leq \limsup _{x \rightarrow 0^{-}} \frac{f(t, x)}{x} \leq b_{0}^{-}(t)
\end{aligned}
$$

uniformly a.e. in $t \in[0, T]$.

Our main result will be achieved by using a topological degree approach on the lines of [16]. A relation between the notions of rotation number and of weighted eigenvalues will be crucial. Multiplicity will be achieved through some information about the number of zeros of the solutions to (1.1).

In the literature, many authors have studied existence and multiplicity of solutions with prescribed nodal properties of problem (1.1) in an asymptotically linear setting.

The first references we wish to quote rely on asymptotically linear problems characterized by the presence of constant bounds in assumptions analogous to $\left(\mathrm{H}^{ \pm}\right)$and $\left(\mathrm{K}^{ \pm}\right)$. The particular case when $a_{\infty}^{+} \equiv a_{\infty}^{-}$and $b_{\infty}^{+} \equiv b_{\infty}^{-}$in $\left(\mathrm{H}^{ \pm}\right)$is usually called "symmetric." In this context, we quote the work [49] by Ubilla where the $p$-Laplacian operator is considered and the work [26] by García-Huidobro and Ubilla where extensions for a wider class of nonhomogeneous differential operators are treated.

In the more general asymmetric context, we refer, among others, to the paper [20] by Dinca-Sanchez. A detailed comparison between our result and the one attained in [20] is developed in the last part of this work (cf. Remark 3.7). We point out that all the articles we have quoted above are written in the spirit of the shooting method and are in the framework of continuous functions. We wish also to mention the paper by Castro and Lazer [11] and the recent contribution attained by Li and Zhou in the work [37], devoted to study with variational methods the existence of multiple nontrivial solutions of $p$-Laplacian-type PDEs. An interesting result is also provided by Esteban in [22].

In the framework of topological methods, we refer to [9], dealing with asymptotically linear asymmetric systems. It is worth noticing that if we restrict ourselves to the scalar symmetric case with constant weights, then [9, Corollary 4.4] and our main result coincide (for a detailed comparison, see [15]).

Other contributions in the symmetric setting are contained in $[4,6,10,23,29,39,40]$ (for PDEs) and in $[38,54]$ (for ODEs).

Multiplicity of solutions of asymptotically, asymmetric semilinear elliptic problems has been widely investigated in the literature. A classical result in this setting is provided by Fučík in [24]. In this direction, we quote, among others, [14, 17, 28, 36] and (for a brief survey) [41]. 
The list of results available in the literature as far as multiplicity of solutions for asymptotically linear problems with weights is concerned is definitely shorter.

Before quoting some recent contributions achieved in this context, we wish to remark that a wider number of authors have studied asymmetric, asymptotically linear problems characterized by assumptions involving indefinite weights in order to get existence of solutions. This kind of problems is usually solved by comparing the given equation in (1.1) with prescribed half-linear problems (which reduce to linear ones in the symmetric case).

This procedure has been adopted in the search for positive solutions to elliptic asymptotically linear or semilinear BVPs (cf., among others, $[3,8,13,18,30,34,43]$ ). In this framework, results have been given on the existence of at least one solution (not necessarily positive) for semilinear boundary value problems both in the symmetric case (see, e.g., [19] by de Figueiredo and Miyagaki as well as [51, 52] by Zhang) and in the asymmetric case (see, e.g., [5] by Arias et al., [21] by Dong, [44] by Reichel and Walter, and [46] by Rynne). We remark that the theory of weighted eigenvalues has been employed in most of the papers quoted above. It is also worth noticing that both in [19] and in [51] additional assumptions are formulated in order to achieve the nontriviality of solutions.

As far as multiplicity of solutions for asymptotically linear problems with weights is concerned, we first mention the works by Sadyrbaev [48] and by Klokov and Sadyrbaev [32] (both generalizing the results in [33]). In these papers the study of an asymmetric boundary value problem is developed through the study of some variation equation. More regularity is required on the nonlinearities. In the asymmetric setting, we wish also to quote the interesting contribution [47] by Rynne. Remarks 3.8 and 3.9 provide more details about these results. Finally, we refer to [16] which handles an asymptotically linear problem of the form (1.1) under symmetric assumptions. For the periodic problems, an analogous result has been attained by Zanini in [50].

It is important to note that the approach used to achieve our result follows the same lines of the one employed in [16]. The present paper extends the results in [16] in two directions: an asymmetric situation is treated and indefinite weights are considered.

In order to present our main result, we now introduce the notion of two-weighted eigenvalues. More precisely, for functions $\varphi, \psi \in \mathrm{L}^{1}([0, T], \mathbb{R})$ such that $\left(\varphi^{+}, \psi^{+}\right) \succ 0$ in $(0, T)$ and for every $\nu \in\{<,>\}$, we consider positively homogeneous asymmetric problems of the form

$$
\begin{gathered}
u^{\prime \prime}(t)+\lambda\left(\varphi(t) u^{+}(t)-\psi(t) u^{-}(t)\right)=0 \\
u(0)=0=u(T), \quad u^{\prime}(0) v 0
\end{gathered}
$$

where $u^{+}:=\max \{u, 0\}, u^{-}:=\max \{-u, 0\}$. We denote by $\lambda^{v}(\varphi, \psi)$ the values of $\lambda$ for which (1.4) admits a solution not identically zero.

In the literature the equation in (1.4) is called half-linear, being positively homogeneous and linear in the cones $u>0$ and $u<0$. A generalized version of the classical Sturm comparison theorems to the half-linear setting will be proved in the following section (see Lemma 2.2). According to this generalization, the existence of a sequence of eigenvalues for problems (1.4) will be ensured. More precisely, the following result (which might be considered of independent interest) will be achieved. 
Theorem 1.1. For every fixed $v \in\{<\rangle$,$\} , problem (1.4) admits a positive monotone in-$ creasing sequence of eigenvalues

$$
0<\lambda_{1}^{\nu}(\varphi, \psi)<\lambda_{2}^{\nu}(\varphi, \psi)<\cdots<\lambda_{j}^{\nu}(\varphi, \psi)<\cdots
$$

Moreover, the half-eigenfunction corresponding to $\lambda_{j}^{\nu}(\varphi, \psi)$ has exactly $j-1$ zeros on $(0, T)$.

An eigenvalue theory for (1.4) has been developed by Alif in [1] and Alif and Gossez in [2] when $\varphi, \psi \in C([0, T], \mathbb{R})$ and $\left(\varphi^{+}, \psi^{+}\right) \succ 0$ in $(0, T)$. We stress the fact that the results achieved in [2] deal with a more general problem, where Fućík-like curves (instead of eigenvalues) are treated. Such results are extended in [1] to the case of $p$-Laplacian operators.

In $[15$, Chapter 4$]$ we treated the hypotheses $\left(\mathrm{H}^{ \pm}\right)$and $\left(\mathrm{K}^{ \pm}\right)$in the particular case where, instead of $\mathrm{L}^{1}$-functions, $C^{1}$-functions are considered. In this framework, we used the eigenvalue theory developed in $[1,2]$.

Now, we are in position to state our main theorem.

Theorem 1.2 (main theorem). Assume that $f:[0, T] \times \mathbb{R} \rightarrow \mathbb{R}$ satisfies the Carathéodory conditions and the assumptions $\left(H^{ \pm}\right)$and $\left(K^{ \pm}\right)$.

Moreover, suppose that there exist $v \in\{<,>\}$ and $n, m \in \mathbb{N}(m \leq n)$ such that either

$$
\lambda_{n}^{v}\left(a_{0}^{+}, a_{0}^{-}\right)<1<\lambda_{m}^{v}\left(b_{\infty}^{+}, b_{\infty}^{-}\right)
$$

or

$$
\lambda_{n}^{\nu}\left(a_{\infty}^{+}, a_{\infty}^{-}\right)<1<\lambda_{m}^{\nu}\left(b_{0}^{+}, b_{0}^{-}\right)
$$

Then, for every $h \in \mathbb{N}$ with $m \leq h \leq n$ problem (1.1) has at least a solution $u_{h, v}$ having exactly $h-1$ zeros in $(0, T)$, with $u_{h, v}^{\prime}(0) \nu 0$.

Clearly, if (1.6) or (1.7) is satisfied for every $\nu \in\{<,>\}$, then, for every $h \in \mathbb{N}$ with $m \leq h \leq n$, Theorem 1.2 guarantees the existence of at least two solutions $u_{h}$ and $v_{h}$ of problem $(1.1)$ with $u_{h}^{\prime}(0)>0$ and $v_{h}^{\prime}(0)<0$ having exactly $h-1$ zeros in $(0, T)$.

This work is organized as follows. Section 2 is devoted to present some preliminary results. More precisely, we first state the abstract topological theorem (Theorem 2.1) providing a first multiplicity result. In the central part of the section, we prove the generalizations of the Sturm theory to the half-linear case. As a consequence, we obtain Theorem 1.1 and a relation between rotation numbers and two-weighted half-eigenvalues (see Lemma 2.6). We conclude Section 2 by recalling the statements of the so-called Elastic lemma. Finally, in Section 3 we obtain the estimates on the rotation numbers which will lead to proving our main theorem. Some comparisons with multiplicity results in the literature are developed in the last part of the paper.

Throughout this paper, we denote by $\mathbb{R}_{0}^{+}$and $\mathbb{R}_{+}=\mathbb{R}_{0}^{+} \cup\{0\}$ the sets of positive and nonnegative real numbers, respectively. Moreover, we define $v_{<}:=(0,-1)$ and $v_{>}:=(0,1)$. 


\section{Preliminary results}

In this section, we introduce the notion of "rotation number" and explain its role when dealing with multiplicity problems.

We consider polar coordinates $(\vartheta, \rho)$, where the angles are counted in the clockwise sense starting from the positive $y$-axis, so that $x=\rho \sin \vartheta, y=\rho \cos \vartheta$. For any continuous curve $\gamma:[0, T] \rightarrow \mathbb{R}^{2} \backslash\{0\}$, we can consider a lifting $\tilde{\gamma}:[0, T] \rightarrow \mathbb{R} \times \mathbb{R}_{0}^{+}$to the $(\vartheta, \rho)$-plane. Setting $\tilde{\gamma}(t)=\left(\vartheta_{\gamma}(t), \rho_{\gamma}(t)\right)$, we have that $\vartheta_{\gamma}$ and $\rho_{\gamma}$ are continuous functions $(\rho(t)=|\gamma(t)|)$ and, moreover, $\vartheta_{\gamma}(t)-\vartheta_{\gamma}(0)$ is independent of the lifting of $\gamma$ which has been considered. Hence, for each $t \in[0, T]$, we can define the rotation number

$$
\operatorname{Rot}(t ; \gamma):=\frac{\vartheta_{\gamma}(t)-\vartheta_{\gamma}(0)}{\pi}
$$

which counts the number of half-turns of the vector $\overrightarrow{0 \gamma(s)}$ as $s$ moves from 0 to $t$.

If the curve $\gamma(t)=\left(u(t), u^{\prime}(t)\right)$ represents a solution $u(\cdot)$ of

$$
u^{\prime \prime}(t)+f(t, u(t))=0
$$

defined on $[0, T]$ and such that $u(t)^{2}+u^{\prime}(t)^{2}>0$ for every $t \in[0, T]$, then we can define the corresponding rotation number. To denote it, we will use the notation $\operatorname{Rot}_{(2.2)}(t ; u)$. Observe that

$$
\operatorname{Rot}_{(2.2)}(t ; u)=\frac{1}{\pi} \int_{0}^{t} \frac{u^{\prime}(t)^{2}+f(t, u(t)) u(t)}{u^{\prime}(t)^{2}+u(t)^{2}} d t
$$

We will now show how some estimates on the rotation number lead to a multiplicity result. The proof of our main result is based on the following theorem which comes from an application of the modified version of the Leray-Schauder continuation theorem stated in $[42]$.

Theorem 2.1. Let $f:[0, T] \times \mathbb{R} \rightarrow \mathbb{R}$ be a Carathéodory function with

$$
f(t, 0) \equiv 0
$$

and such that $u(\cdot) \equiv 0$ is the unique solution of (2.2) satisfying $u\left(t_{0}\right)=u^{\prime}\left(t_{0}\right)=0$ for some $t_{0} \in \mathbb{R}$. Assume also that for each $z_{0} \in \mathbb{R}^{2} \backslash\{0\}$, all the solutions of (2.2) with $\left(u(0), u^{\prime}(0)\right)=$ $z_{0}$ can be defined on $[0, T]$.

Suppose that there are a positive integer $j$ and two positive numbers $r<R$ such that

$$
\operatorname{Rot}_{(2.2)}(T ; u)>j \quad(\text { resp. },<j)
$$

for each solution $u$ of (2.2) with $u(0)=0,\left|u^{\prime}(0)\right|=r, u^{\prime}(0) \nu 0$, as well as

$$
\operatorname{Rot}_{(2.2)}(T ; u)<j \quad(\text { resp., }>j)
$$

for each solution $u$ of (2.2) with $u(0)=0,\left|u^{\prime}(0)\right|=R, u^{\prime}(0) v 0$. Then, there exists at least one solution $u_{j}$ of (1.1) with $u_{j}^{\prime}(0) v 0$ having exactly $j-1$ zeros in $(0, T)$. 
For the proof, we refer to [16].

In order to prove our main result, we are first interested in providing a generalization of the Sturm comparison theorems for half-linear asymmetric two-weighted equations of the form

$$
u^{\prime \prime}(t)+\lambda\left(\varphi(t) u^{+}(t)-\psi(t) u^{-}(t)\right)=0
$$

where $\varphi, \psi \in \mathrm{L}^{1}([0, T], \mathbb{R})$ and $\lambda \in \mathbb{R}_{0}^{+}$.

We introduce now a simpler notation for rotation numbers of solutions to (2.7). Considering $v \in S^{1}$ and $k \in \mathbb{R}$, it is well known that the Cauchy problem

$$
\begin{gathered}
u^{\prime \prime}(t)+\lambda \varphi(t) u^{+}(t)-\lambda \psi(t) u^{-}(t)=0, \\
\left(u(0), u^{\prime}(0)\right)=k v
\end{gathered}
$$

admits a unique solution, denoted by $u(\cdot ; k v)$. Furthermore, the rotation number of $u(\cdot ; k v)$ is well defined whenever $k \neq 0$, since in this case it is easy to show that $u(t ; k v)^{2}+$ $u^{\prime}(t ; k v)^{2}>0$ for every $t \in[0, T]$. As $u(t ; k v)=k u(t ; v)$, one can also see that the rotation number is independent on $k$ for $k \neq 0$. Hence, it is not restrictive to work with $k=1$.

For this reason, we will set

$$
\operatorname{Rot}_{\lambda \varphi, \lambda \psi}(t ; v):=\operatorname{Rot}_{(2.7)}(t ; u(\cdot ; v)) .
$$

In order to discuss the dependence of the rotation numbers upon the coefficients of (2.7), we rewrite problem (2.7) in the equivalent form

$$
u^{\prime}(t)=\lambda z(t), \quad z^{\prime}(t)=-\varphi(t) u^{+}(t)+\psi(t) u^{-}(t) .
$$

We introduce now the polar coordinates $(\theta, r) \in \mathbb{R} \times \mathbb{R}_{0}^{+}$by setting $u(t)=r(t) \sin \theta(t)$, $z(t)=u^{\prime}(t) / \lambda=r(t) \cos \theta(t)$. In particular, the angular coordinate $\theta:=\theta_{\lambda ; \varphi, \psi}$ satisfies

$$
\theta^{\prime}(t)= \begin{cases}\lambda \cos ^{2} \theta(t)+\varphi(t) \sin ^{2} \theta(t) & \text { if } \sin \theta(t) \geq 0 \\ \lambda \cos ^{2} \theta(t)+\psi(t) \sin ^{2} \theta(t) & \text { if } \sin \theta(t)<0\end{cases}
$$

As a first step, we generalize the classical Sturm comparison theorem to the asymmetric, indefinite case. This is of fundamental importance for the proof of the existence of a sequence of weighted eigenvalues for (1.4). We refer to Theorem 2.5 for more details.

Lemma 2.2. Let $\varphi_{i}, \psi_{i} \in \mathrm{L}^{1}([0, T], \mathbb{R}), \lambda_{i} \in \mathbb{R}_{0}^{+}(i=1,2)$ such that $\left(\varphi_{1}, \psi_{1}\right) \geq\left(\varphi_{2}, \psi_{2}\right)$ for a.e. $t \in[0, T]$ and $\lambda_{1} \geq \lambda_{2}$. According to the notation $\theta_{i}:=\theta_{\lambda_{i} ; \varphi_{i}, \psi_{i}}$, it is supposed that $\theta_{1}(0)=$ $\theta_{2}(0)$. Then,

$$
\theta_{1}(t) \geq \theta_{2}(t) \quad \forall t \in[0, T]
$$

Moreover, if $\left(\varphi_{1}, \psi_{1}\right) \succ\left(\varphi_{2}, \psi_{2}\right)$ or if $\cos \theta_{1}(0) \neq 0$ and $\lambda_{1}>\lambda_{2}$, then

$$
\theta_{1}(0)=\theta_{2}(0) \Longrightarrow \theta_{1}(T)>\theta_{2}(T) \text {. }
$$


Slight variants of the first part of this lemma have been proved in $[21,44]$. In what follows, we develop a complete proof, using different arguments.

Proof. Following a classical procedure (see [12]), we consider $\theta:=\theta_{2}-\theta_{1}$. It is easy to show that

$$
\theta^{\prime}=\left(\sin ^{2} \theta_{2}-\sin ^{2} \theta_{1}\right)\left(g_{1}-\lambda_{1}\right)+\cos ^{2} \theta_{2}\left(\lambda_{2}-\lambda_{1}\right)+\sin ^{2} \theta_{2}\left(g_{2}-g_{1}\right)
$$

where

$$
g_{i}(t):= \begin{cases}\varphi_{i}(t) & \text { if } \sin \theta_{i}(t)>0 \\ \psi_{i}(t) & \text { if } \sin \theta_{i}(t)<0\end{cases}
$$

Notice that the right-hand side of (2.14) is defined almost everywhere, since from (2.11) the function $\sin \theta_{i}$ has only isolated zeros in $t$.

We first assume that $\left(\varphi_{1}, \psi_{1}\right) \geq\left(\varphi_{2}, \psi_{2}\right)$ for a.e. $t \in[0, T]$ and $\lambda_{1} \geq \lambda_{2}$.

We first prove the nonpositivity of $\left(g_{2}-g_{1}\right)$ a.e. in a subinterval of $[0, T]$. From (2.11), we obtain that $\theta_{i}$ crosses each line $k \pi$ only from below, when $k \in \mathbb{Z}$ and $i \in\{1,2\}$. Thus, taking into account that $\theta_{1}(0)=\theta_{2}(0)$, one can deduce the existence of $S \in(0, T)$ such that $\sin \theta_{1}(t) \cdot \sin \theta_{2}(t)>0$ for every $t \in(0, S)$. This means that $g_{1}$ and $g_{2}$ are comparable in the interval $\mathrm{I}=(0, S)$ and, precisely, $\left(g_{2}(t)-g_{1}(t)\right) \leq 0$ for a.e. $t \in \mathrm{I}$.

Thus, by assumptions and from equality (2.14), we get

$$
\theta^{\prime}(t)-f(t) \theta(t) \leq 0 \quad \text { for a.e. } t \in \mathrm{I} \text {, where } f:=\frac{\left(\sin ^{2} \theta_{2}-\sin ^{2} \theta_{1}\right)}{\theta_{2}-\theta_{1}}\left(g_{1}-\lambda_{1}\right) \text {. }
$$

Note that $f \in \mathrm{L}^{1}([0, T], \mathbb{R})$. If we define $F(t):=\int_{t}^{T} f(s) d s$, we obtain

$$
e^{F(t)} \theta(t) \leq e^{F(0)} \theta(0)=0 \quad \forall t \in \overline{\mathrm{I}}:=[0, S] .
$$

In particular, $\theta_{1}(t) \geq \theta_{2}(t)$ for all $t \in \overline{\mathrm{I}}$.

We are now interested in extending the above inequality in the whole interval $[0, T]$. We assume, by contradiction, that there exists $\widetilde{R} \in(S, T)$ such that $\theta_{1}(\widetilde{R})<\theta_{2}(\widetilde{R})$. By the continuity of $\theta(\cdot)$, it is possible to find a constant $R \in[S, \widetilde{R})$ such that $\theta(R)=0$ and $\theta(t)>$ 0 for every $t \in(R, \widetilde{R}]$. Arguing exactly as before, we can infer the existence of a nonempty interval of the form $\left[R, S^{*}\right]$ where the function $\theta$ is nonpositive, a contradiction.

Assume now that either $\left(\varphi_{1}, \psi_{1}\right) \succ\left(\varphi_{2}, \psi_{2}\right)$ or $\cos \theta_{1}(0) \neq 0$ and $\lambda_{1}>\lambda_{2}$.

The previous step of the proof guarantees that $\theta_{1}(t) \geq \theta_{2}(t)$ for every $t \in[0, T]$.

We first claim that the existence of a constant $P \in(0, T]$ such that $\theta_{1}(P)>\theta_{2}(P)$ implies that $\theta_{1}(t)>\theta_{2}(t)$ for every $t \in[P, T]$.

By contradiction, assume that there exists $\bar{R} \in(P, T]$ such that $\theta(\bar{R})=0$ and $\theta(t)<0$ for every $t \in[P, \bar{R})$. In particular, $\sin \theta_{1}(s) \cdot \sin \theta_{2}(s)>0$ for every $s \in[R, \bar{R})$, for a suitable $R \in[P, \bar{R})$. As before, we can verify that

$$
e^{F(t)} \theta(t) \leq e^{F(R)} \theta(R)<0 \quad \forall t \in[R, \bar{R}],
$$

a contradiction with $\theta(\bar{R})=0$. 
964 Multiplicity results for asymmetric BVPs with weights

Consider now the case $\lambda_{1}>\lambda_{2}$ and $\cos \theta_{1}(0)=\cos \theta_{2}(0) \neq 0$. From (2.14), we immediately get the existence of an interval $J=\left(0, P^{*}\right]$ where $\theta^{\prime}-f \theta$ is a.e. negative. This implies that

$$
\left(e^{F(t)} \theta(t)\right)^{\prime}<0 \quad \text { for a.e. } t \in J
$$

and, consequently, $\theta_{1}(t)>\theta_{2}(t)$ for every $t \in J$. The thesis follows by the previous claim.

Finally, consider the case $\left(\varphi_{1}, \psi_{1}\right) \succ\left(\varphi_{2}, \psi_{2}\right)$. By assumption, there exists a nonempty interval $I=[a, b] \subset[0, T]$ such that $\varphi_{1}(t)>\varphi_{2}(t)$ and $\psi_{1}(t)>\psi_{2}(t)$ for a.e. $t \in I$. We suppose, by contradiction, that $\theta_{1}(T)=\theta_{2}(T)$. Hence, by an application of the previous claim, we deduce that $\theta_{1}(t)=\theta_{2}(t)$ for every $t \in[0, T]$. From a suitable choice of the constants $a, b$, we can also show that $\sin \theta_{1}(t) \cdot \sin \theta_{2}(t)>0$ for every $t \in I$ and, consequently, $\theta^{\prime}(t)-f(t) \theta(t)<0$ a.e. in $I$. Arguing as above, we can conclude that $\theta_{1}(t)>\theta_{2}(t)$ for every $t \in I$, a contradiction.

Consider a constant $c \neq(1+2 k) \pi / 2$, for all $k \in \mathbb{Z}$. Lemma 2.2 ensures that the function $\theta_{\lambda ; \varphi, \psi}(T)$ is strictly increasing with respect to $\lambda$, under the initial condition $\theta_{\lambda ; \varphi, \psi}(0)=c$. We remark that the proof of Theorem 2.5 is based on this monotonicity result.

Now, we state a corollary of Lemma 2.2, which follows from the monotonicity of $\theta$ with respect to the weights.

Lemma 2.3. Let $\varphi_{i}, \psi_{i} \in \mathrm{L}^{1}([0, T], \mathbb{R})(i=1,2)$ such that $\left(\varphi_{1}, \psi_{1}\right) \geq\left(\varphi_{2}, \psi_{2}\right)$ for a.e. $t \in$ $[0, T]$. Then,

$$
\operatorname{Rot}_{\varphi_{1}, \psi_{1}}(t ; v) \geq \operatorname{Rot}_{\varphi_{2}, \psi_{2}}(t ; v), \quad \forall t \in[0, T], \forall v \in S^{1} .
$$

If, moreover, $\left(\varphi_{1}, \psi_{1}\right) \succ\left(\varphi_{2}, \psi_{2}\right)$, then

$$
\operatorname{Rot}_{\varphi_{1}, \psi_{1}}(T ; v)>\operatorname{Rot}_{\varphi_{2}, \psi_{2}}(T ; v), \quad \forall v \in S^{1} .
$$

For an analogous result we refer to [53, Lemma 2.1].

By an application of Lemma 2.3, we obtain the following.

Lemma 2.4. Let $\varphi, \psi \in \mathrm{L}^{1}([0, T], \mathbb{R})$ and $v \in S^{1}$. For each $\varepsilon>0$, there is $\delta>0$ such that, for all the functions $\varphi_{*}, \psi_{*} \in \mathrm{L}^{1}([0, T], \mathbb{R})$ satisfying

$$
\varphi_{*}(t) \geq \varphi(t)-\delta, \quad \psi_{*}(t) \geq \psi(t)-\delta, \quad \text { for a.e. } t \in[0, T],
$$

it follows that

$$
\operatorname{Rot}_{\varphi_{*}, \psi_{*}}(t ; v)>\operatorname{Rot}_{\varphi, \psi}(t ; v)-\varepsilon, \quad \forall t \in[0, T] .
$$

Analogously, for each $\varepsilon>0$ there is $\delta>0$ such that, for all the functions $\varphi_{*}, \psi_{*} \in \mathrm{L}^{1}([0, T], \mathbb{R})$ satisfying

$$
\varphi_{*}(t) \leq \varphi(t)+\delta, \quad \psi_{*}(t) \leq \psi(t)+\delta, \quad \text { for a.e. } t \in[0, T],
$$

it follows that

$$
\operatorname{Rot}_{\varphi_{*}, \psi_{*}}(t ; v)<\operatorname{Rot}_{\varphi, \psi}(t ; v)+\varepsilon, \quad \forall t \in[0, T] .
$$


We remark that this lemma extends [16, Lemma 3.3] to the half-linear context. Hence, to prove Lemma 2.4 it is possible to use an argument similar to the one used in [16].

Proof. In what follows, we will provide a proof only of the first part of the lemma. The second part can be proved in an analogous way (just reverse the inequalities).

Let $\varepsilon>0$ be fixed and suppose that $\varphi, \psi, \varphi_{*}, \psi_{*}$ satisfy (2.22) for some $\delta>0$. By Lemma 2.3, we know that $\operatorname{Rot}_{\varphi_{*}, \psi_{*}}(t ; v) \geq \operatorname{Rot}_{\varphi-\delta, \psi-\delta}(t ; v)$ for every $t \in[0, T]$. Hence, to prove the result, it will be sufficient to check that for a suitable choice of $\delta>0$ (sufficiently small), it follows that

$$
\operatorname{Rot}_{\varphi-\delta, \psi-\delta}(t ; v)>\operatorname{Rot}_{\varphi, \psi}(t ; v)-\varepsilon \quad \forall t \in[0, T]
$$

Assume, by contradiction, that for each $n$ there is $t_{n} \in[0, T]$ such that

$$
\operatorname{Rot}_{\varphi-1 / n, \psi-1 / n}\left(t_{n} ; v\right) \leq \operatorname{Rot}_{\varphi, \psi}\left(t_{n} ; v\right)-\varepsilon
$$

Without loss of generality, we can also suppose that $t_{n} \rightarrow \tau \in[0, T]$, so that

$$
\limsup _{n \rightarrow+\infty} \operatorname{Rot}_{\varphi-1 / n, \psi-1 / n}\left(t_{n} ; v\right) \leq \operatorname{Rot}_{\varphi, \psi}(\tau ; v)-\varepsilon .
$$

Let $u_{n}(\cdot), u(\cdot)$ be, respectively, the solutions of

$$
u^{\prime \prime}+\left(\varphi(t)-\frac{1}{n}\right) u^{+}(t)-\left(\psi(t)-\frac{1}{n}\right) u^{-}(t)=0
$$

and of (2.7), with

$$
\left(u_{n}(0), u_{n}^{\prime}(0)\right)=\left(u(0), u^{\prime}(0)\right)=v .
$$

By the continuous dependence of the solutions from the equations data, which is valid also in case of Carathéodory assumptions [27], we know that $u_{n} \rightarrow u$ in the $C^{1}$-norm, uniformly on $[0, T]$. Hence,

$$
\begin{aligned}
& \operatorname{Rot}_{\varphi-1 / n, \psi-1 / n}\left(t_{n} ; v\right) \\
& =\frac{1}{\pi} \int_{0}^{t_{n}} \frac{u_{n}^{\prime}(t)^{2}+(\varphi(t)-1 / n) u_{n}^{+}(t) u_{n}(t)-(\psi(t)-1 / n) u_{n}^{-}(t) u_{n}(t)}{u_{n}^{\prime}(t)^{2}+u_{n}(t)^{2}} d t \\
& \longrightarrow \frac{1}{\pi} \int_{0}^{\tau} \frac{u^{\prime}(t)^{2}+\left(\varphi(t) u^{+}(t)-\psi(t) u^{-}(t)\right) u(t)}{u^{\prime}(t)^{2}+u(t)^{2}} d t=\operatorname{Rot}_{\varphi, \psi}(\tau ; v),
\end{aligned}
$$

which is a contradiction with (2.28). Then, (2.26) is achieved and the proof is complete.

Our aim consists now in generalizing the comparison results contained in Lemma 2.4 to asymptotically linear equations (see Lemma 3.1). To this purpose we need to exhibit a relation between weighted eigenvalues and rotation numbers of solutions to the following 
half-linear Cauchy problems:

$$
\begin{gathered}
u^{\prime \prime}(t)+\lambda\left(\varphi(t) u^{+}(t)-\psi(t) u^{-}(t)\right)=0 \\
\left(u(0), u^{\prime}(0)\right)=v_{v}
\end{gathered}
$$

where $\nu \in\{<,>\}$ and $\varphi, \psi \in \mathrm{L}^{1}([0, T], \mathbb{R})$ with $\left(\varphi^{+}, \psi^{+}\right) \succ(0,0)$ on $(0, T)$. According to the notation previously introduced, we recall that $v_{<}:=(0,-1)$ and $v_{>}:=(0,1)$.

We first prove that there exists a positive sequence of weighted eigenvalues for halflinear problems of the form (1.4). More precisely, we have the following.

Theorem 2.5. For every fixed $v \in\{<\rangle$,$\} , problem (1.4) admits a positive monotone in-$ creasing sequence of eigenvalues

$$
0<\lambda_{1}^{\nu}(\varphi, \psi)<\lambda_{2}^{\nu}(\varphi, \psi)<\cdots<\lambda_{j}^{\nu}(\varphi, \psi) \longrightarrow+\infty \quad \text { as } j \longrightarrow+\infty .
$$

Moreover, the half-eigenfunction corresponding to $\lambda_{j}^{\nu}(\varphi, \psi)$ has exactly $j-1$ zeros on $(0, T)$. Idea of the proof. We omit the details of the proof since the procedure followed is classical (see, e.g., [12]).

To represent a solution $\left(u(t), u^{\prime}(t)\right)$ of $(2.32)$, we use the polar coordinates $\left(\vartheta_{\lambda}^{\gamma}(t), \rho_{\lambda}^{\nu}(t)\right)=$ $(\vartheta, \rho)$ adopted in order to define the rotation numbers, so that $u=\rho \sin \vartheta, u^{\prime}=\rho \cos \vartheta$. Without loss of generality, we assume the validity of the initial condition $\vartheta_{\lambda}^{\nu}(0)=\vartheta_{\nu}^{0}$, where $\vartheta_{>}^{0}=0$ and $\vartheta_{<}^{0}=\pi$.

It is possible to deduce the continuity of the function $\lambda \mapsto \vartheta_{\lambda}^{\nu}(T)$, defined on $\mathbb{R}$. Moreover, one can show that

$$
\lim _{\lambda \rightarrow+\infty} \vartheta_{\lambda}^{v}(T)=+\infty, \quad \vartheta_{0}^{v}(T) \in\left(\vartheta_{\nu}^{0}, \vartheta_{\nu}^{0}+\frac{\pi}{2}\right)
$$

Hence, for every $j \in \mathbb{N}$ there exists at least a positive value of $\lambda$ for which $\vartheta_{\lambda}^{\nu}(T)=\vartheta_{\nu}^{0}+j \pi$. We denote such an eigenvalue by $\lambda_{j}^{v}$.

It only remains to prove its uniqueness.

This will be achieved by applying Lemma 2.2 , where another angular function $\theta_{\lambda ; \varphi, \psi}$ (different from $\vartheta_{\lambda}^{\nu}$ ) is treated. We denote by $\theta_{\lambda ; \varphi, \psi ; \nu}=\theta_{\lambda ; \nu}$ the solution of (2.11) satisfying the initial condition $\theta_{\lambda ; \nu}(0)=\vartheta_{\nu}^{0}$.

Lemma 2.2 guarantees that the map $\lambda \mapsto \theta_{\lambda ; \nu}(T)$ is strictly increasing in $\mathbb{R}_{0}^{+}$.

It is then sufficient to prove the validity of the following relations for $k \in \mathbb{N}$ :

$$
\begin{gathered}
\vartheta_{\lambda}^{\nu}(t)=\vartheta_{\nu}^{0}+\frac{k \pi}{2} \Longleftrightarrow \theta_{\lambda ; \nu}(t)=\vartheta_{\nu}^{0}+\frac{k \pi}{2}, \\
\vartheta_{\lambda}^{\nu}(t)-\vartheta_{\nu}^{0} \in\left(\frac{k \pi}{2}, \frac{(k+1) \pi}{2}\right) \Longleftrightarrow \theta_{\lambda ; \nu}(t)-\vartheta_{\nu}^{0} \in\left(\frac{k \pi}{2}, \frac{(k+1) \pi}{2}\right) .
\end{gathered}
$$

Indeed, by combining these relations with Lemma 2.2, we can deduce that also $\vartheta_{(.)}^{v}(T)$ crosses each line $\vartheta_{v}^{0}+j \pi$ exactly once. The uniqueness of the $j$ th eigenvalue $\lambda_{j}^{v}=\lambda_{j}^{v}(\varphi, \psi)$ is consequently ensured. Moreover, the corresponding half-eigenfunction has exactly $j-$ 1 zeros on $(0, T)$. 
To complete the proof, we observe that the validity of (2.35) easily follows from the definition of the angular functions and, in particular, from the equalities

$$
\begin{gathered}
\rho_{\lambda}^{\nu}(t) \sin \vartheta_{\lambda}^{\nu}(t)=u(t)=r_{\lambda ; \nu}(t) \sin \theta_{\lambda ; \nu}(t), \\
\rho_{\lambda}^{\nu}(t) \cos \vartheta_{\lambda}^{\nu}(t)=u^{\prime}(t)=\lambda r_{\lambda ; \nu}(t) \cos \theta_{\lambda ; \nu}(t),
\end{gathered}
$$

where $\left(u(t), u^{\prime}(t)\right)$ represents a solution of $(2.32)$ and $\lambda, \rho_{\lambda}^{\nu}(\cdot), r_{\lambda ; \nu}(\cdot) \in \mathbb{R}_{0}^{+}$.

Taking into account Lemma 2.2, the angular relations (2.35), and the characterization of the eigenvalues established by Theorem 2.5 , we immediately deduce the validity of the following relation between rotation numbers and weighted eigenvalues.

Lemma 2.6. Consider the Cauchy problem (2.32) where, as usual, $v \in\{<,>\}$ and $\varphi, \psi \in$ $\mathrm{L}^{1}([0, T], \mathbb{R})$ with $\left(\varphi^{+}, \psi^{+}\right) \succ(0,0)$ on $(0, T)$. Then,

$$
\begin{aligned}
& \operatorname{Rot}_{\lambda \varphi, \lambda \psi}\left(T ; v_{\nu}\right)=j \Longleftrightarrow \lambda=\lambda_{j}^{\nu}(\varphi, \psi), \\
& \operatorname{Rot}_{\lambda \varphi, \lambda \psi}\left(T ; v_{\nu}\right)>j \Longleftrightarrow \lambda>\lambda_{j}^{\nu}(\varphi, \psi), \\
& \operatorname{Rot}_{\lambda \varphi, \lambda \psi}\left(T ; v_{\nu}\right)<j \Longleftrightarrow \lambda<\lambda_{j}^{\nu}(\varphi, \psi) .
\end{aligned}
$$

Under continuous assumptions on the weights, Lemma 2.6 consists of a reformulation of some results achieved in [2] in terms of the rotation number.

The last part of this section is devoted to recalling the well-known result called Elastic lemma (cf. [25]). We consider the problem

$$
v^{\prime \prime}+h(t, v(t))=0
$$

where $h:\left[t_{1}, t_{2}\right] \times \mathbb{R} \rightarrow \mathbb{R}$ satisfies the Carathéodory conditions and there exist two functions $C, m \in \mathrm{L}^{1}\left(\left[t_{1}, t_{2}\right], \mathbb{R}_{+}\right)$such that

$$
|h(t, x)| \leq C(t)|x|+m(t)
$$

for every $x \in \mathbb{R}$ and for a.e. $t \in\left[t_{1}, t_{2}\right]$, with $t_{1}, t_{2} \in \mathbb{R}$ such that $t_{1}<t_{2}$.

Lemma 2.7. For every $R_{1}>0$, there exists $R_{2}>R_{1}$ such that for every solution $v$ of the problem (2.38) with

$$
\min _{t \in\left[t_{1}, t_{2}\right]}\left|\left(v(t), v^{\prime}(t)\right)\right| \leq R_{1}
$$

it follows that

$$
\left|\left(v(t), v^{\prime}(t)\right)\right| \leq R_{2} \quad \forall t \in\left[t_{1}, t_{2}\right] .
$$

Consider again the problem (2.38), where $h:[0, T] \times \mathbb{R} \rightarrow \mathbb{R}$ satisfies the Carathéodory conditions. Suppose now the existence of a function $C \in \mathrm{L}^{1}\left([0, T], \mathbb{R}_{+}\right)$and of a constant $\delta>0$ such that

$$
|h(t, x)| \leq C(t)|x| \quad \forall|x| \leq \delta \text {, for a.e. } t \in[0, T]
$$


968 Multiplicity results for asymmetric BVPs with weights

Under these hypotheses, a dual situation with respect to Lemma 2.7 occurs at zero and it can be expressed by the following lemma.

LEMMA 2.8. For every $\rho_{1}>0$ there exists $\rho_{2}<\rho_{1}$ such that for every solution $v$ of the problem (2.38) with

$$
\max _{t \in[0, T]}\left|\left(v(t), v^{\prime}(t)\right)\right|>\rho_{1}
$$

it follows that

$$
\left|\left(v(t), v^{\prime}(t)\right)\right|>\rho_{2} \quad \forall t \in[0, T] .
$$

This lemma can be seen as a consequence of Lemma 2.7.

\section{The main result}

As a first step, we establish a relation between the rotation number of solutions to equation (2.2) and the rotation number of solutions to suitable half-eigenvalue problems, in the spirit of extending the comparison Lemma 2.4 to the framework of nonlinear equations. In Lemma 3.1, we will implicitly assume that the solutions of (2.2) we consider are defined in $[0, T]$ and are such that $\left(u(t), u^{\prime}(t)\right) \neq 0$, for all $t \in[0, T]$. In the sequel, we will confine our applications to nonlinear equations which are asymptotically linear at zero and at infinity and, therefore, all the solutions (but the trivial one) of (2.2) will satisfy such assumptions.

Lemma 3.1. Let $f:[0, T] \times \mathbb{R} \rightarrow \mathbb{R}$ be a Carathéodory function and let $\varphi, \psi \in \mathrm{L}^{1}([0, T], \mathbb{R})$ be such that

$$
\liminf _{x \rightarrow+\infty} \frac{f(t, x)}{x} \geq \varphi(t), \quad \liminf _{x \rightarrow-\infty} \frac{f(t, x)}{x} \geq \psi(t), \quad \text { for a.e. } t \in[0, T],
$$

hold uniformly in $t$. Then, for each $\varepsilon>0$, there is $R_{\varepsilon}>0$ such that for each solution $u(\cdot)$ of (2.2) with $\left|\left(u(t), u^{\prime}(t)\right)\right| \geq R_{\varepsilon}$ for all $t \in[0, T]$, it follows that

$$
\operatorname{Rot}_{(2.2)}(t ; u) \geq \operatorname{Rot}_{\varphi, \psi}(t ; v)-\varepsilon, \quad \forall t \in[0, T], \text { with } v=\frac{\left(u(0), u^{\prime}(0)\right)}{\left|\left(u(0), u^{\prime}(0)\right)\right|}
$$

Analogously, if

$$
\limsup _{x \rightarrow+\infty} \frac{f(t, x)}{x} \leq \varphi(t), \quad \limsup _{x \rightarrow-\infty} \frac{f(t, x)}{x} \leq \psi(t), \quad \text { for a.e. } t \in[0, T],
$$

hold uniformly in $t$, then, for each $\varepsilon>0$ there is $R_{\varepsilon}>0$ such that for each solution $u(\cdot)$ of (2.2) with $\left|\left(u(t), u^{\prime}(t)\right)\right| \geq R_{\varepsilon}$ for all $t \in[0, T]$, it follows that

$$
\operatorname{Rot}_{(2.2)}(t ; u) \leq \operatorname{Rot}_{\varphi, \psi}(t ; v)+\varepsilon, \quad \forall t \in[0, T], \text { with } v=\frac{\left(u(0), u^{\prime}(0)\right)}{\left|\left(u(0), u^{\prime}(0)\right)\right|}
$$


Furthermore, if it is assumed that

$$
\liminf _{x \rightarrow 0^{+}} \frac{f(t, x)}{x} \geq \varphi(t), \quad \liminf _{x \rightarrow 0^{-}} \frac{f(t, x)}{x} \geq \psi(t), \quad \text { for a.e. } t \in[0, T],
$$

hold uniformly in $t$, then, for each $\varepsilon>0$ there is $r_{\varepsilon}>0$ such that for each solution $u(\cdot)$ of (2.2) with $0<\left|\left(u(t), u^{\prime}(t)\right)\right| \leq r_{\varepsilon}$ for all $t \in[0, T]$, it follows that

$$
\operatorname{Rot}_{(2.2)}(t ; u) \geq \operatorname{Rot}_{\varphi, \psi}(t ; v)-\varepsilon \quad \forall t \in[0, T], \text { with } v=\frac{\left(u(0), u^{\prime}(0)\right)}{\left|\left(u(0), u^{\prime}(0)\right)\right|} .
$$

Analogously, if

$$
\limsup _{x \rightarrow 0^{+}} \frac{f(t, x)}{x} \leq \varphi(t), \quad \limsup _{x \rightarrow 0^{-}} \frac{f(t, x)}{x} \leq \psi(t), \quad \text { for a.e. } t \in[0, T],
$$

hold uniformly in $t$, then, for each $\varepsilon>0$ there is $r_{\varepsilon}>0$ such that for each solution $u(\cdot)$ of (2.2) with $0<\left|\left(u(t), u^{\prime}(t)\right)\right| \leq r_{\varepsilon}$ for all $t \in[0, T]$, it follows that

$$
\operatorname{Rot}_{(2.2)}(t ; u) \leq \operatorname{Rot}_{\varphi, \psi}(t ; v)+\varepsilon \quad \forall t \in[0, T], \text { with } v=\frac{\left(u(0), u^{\prime}(0)\right)}{\left|\left(u(0), u^{\prime}(0)\right)\right|} .
$$

The above lemma represents a generalization of [16, Lemma 3.4] to the asymmetric setting.

Proof. Assume (3.1) and let $\varepsilon>0$ be fixed. By Lemma 2.4, there is $\delta>0$ such that

$$
\operatorname{Rot}_{\varphi-\delta, \psi-\delta}(t ; v) \geq \operatorname{Rot}_{\varphi, \psi}(t, v)-\frac{\varepsilon}{2}, \quad \forall t \in[0, T], \forall v \in S^{1}
$$

For such a $\delta$, using (3.1) and the Carathéodory assumptions, we can find a function $\ell=\ell_{\delta} \in \mathrm{L}^{1}\left([0, T], \mathbb{R}_{+}\right)$such that

$$
f(t, x) x \geq(\varphi(t)-\delta) x^{+} x-(\psi(t)-\delta) x^{-} x-\ell(t), \quad \forall x \in \mathbb{R} \text {, for a.e. } t \in[0, T],
$$

where $x^{+}:=\max \{x, 0\}$ and $x^{-}:=\max \{-x, 0\}$. Now, assume by contradiction that the claimed consequence of (3.1) is not true. This means that for each $n$ there is a solution $u_{n}$ of (2.2) defined on $[0, T]$ with $\rho_{n}(t)=\left|\left(u_{n}(t), u_{n}^{\prime}(t)\right)\right| \geq n$ for all $t \in[0, T]$ and such that, for some $t_{n} \in[0, T]$,

$$
\operatorname{Rot}_{(2.2)}\left(t_{n} ; u_{n}\right)<\operatorname{Rot}_{\varphi, \psi}\left(t_{n} ; v_{n}\right)-\varepsilon
$$

where

$$
v_{n}=\frac{\left(u_{n}(0), u_{n}^{\prime}(0)\right)}{\left|\left(u_{n}(0), u_{n}^{\prime}(0)\right)\right|}=\left(\sin \left(\alpha_{n}\right), \cos \left(\alpha_{n}\right)\right) .
$$

Without loss of generality, it is also possible to assume that $t_{n} \rightarrow \tau \in[0, T]$ and $v_{n} \rightarrow w=$ $(\sin (\alpha), \cos (\alpha)) \in S^{1}$ with $\alpha_{n} \rightarrow \alpha$. Then, passing to the upper limit on the above equation 
970 Multiplicity results for asymmetric BVPs with weights

and using the continuity of $\operatorname{Rot}_{\varphi, \psi}(\cdot ; \cdot)$ in $[0, T] \times S^{1}$, we obtain

$$
\limsup _{n \rightarrow \infty} \operatorname{Rot}_{(2.2)}\left(t_{n} ; u_{n}\right) \leq \operatorname{Rot}_{\varphi, \psi}(\tau ; w)-\varepsilon .
$$

Next, we use the polar coordinates $\left(\vartheta_{n}(t), \rho_{n}(t)\right)$ to represent $\left(u_{n}(t), u_{n}^{\prime}(t)\right)$. We obtain that

$$
\begin{aligned}
\vartheta_{n}^{\prime}(t) & =\frac{u_{n}^{\prime}(t)^{2}+u_{n}(t) f\left(t, u_{n}(t)\right)}{u_{n}^{\prime}(t)^{2}+u_{n}(t)^{2}} \\
& \geq \cos ^{2} \vartheta_{n}(t)+(\varphi(t)-\delta)\left(\sin \vartheta_{n}(t)\right)^{+} \sin \vartheta_{n}(t)-(\psi(t)-\delta)\left(\sin \vartheta_{n}(t)\right)^{-} \sin \vartheta_{n}(t)-\frac{\ell(t)}{\rho_{n}^{2}(t)} \\
& \geq \cos ^{2} \vartheta_{n}(t)+(\varphi(t)-\delta)\left(\sin \vartheta_{n}(t)\right)^{+} \sin \vartheta_{n}(t)-(\psi(t)-\delta)\left(\sin \vartheta_{n}(t)\right)^{-} \sin \vartheta_{n}(t)-\frac{\ell(t)}{n}
\end{aligned}
$$

holds for almost every $t \in[0, T]$. By a result on differential inequalities [35] we know that

$$
\operatorname{Rot}_{(2.2)}\left(t ; u_{n}\right)=\frac{\vartheta_{n}(t)-\vartheta_{n}(0)}{\pi}=\frac{\vartheta_{n}(t)-\alpha_{n}}{\pi} \geq \frac{\theta_{n}(t)-\alpha_{n}}{\pi},
$$

where $\theta_{n}$ is the solution of

$$
\begin{gathered}
\theta^{\prime}=\cos ^{2} \theta+(\varphi(t)-\delta)(\sin \theta)^{+} \sin \theta-(\psi(t)-\delta)(\sin \theta)^{-} \sin \theta-\frac{\ell(t)}{n}, \\
\theta(0)=\alpha_{n} .
\end{gathered}
$$

By continuous dependence (with respect to $\mathrm{L}^{1}$-perturbations of the vector field), we know that, as $n \rightarrow \infty, \theta_{n} \rightarrow \bar{\theta}$, uniformly on $[0, T], \bar{\theta}(\cdot)$ being the solution of

$$
\begin{gathered}
\theta^{\prime}(t)=\cos ^{2} \theta(t)+(\varphi(t)-\delta)(\sin \theta(t))^{+} \sin \theta(t)-(\psi(t)-\delta)(\sin \theta(t))^{-} \sin \theta(t), \\
\theta(0)=\alpha .
\end{gathered}
$$

In particular, by uniform convergence, we have that $\theta_{n}\left(t_{n}\right) \rightarrow \bar{\theta}(\tau)$ for $n \rightarrow \infty$. Thus, we can conclude that

$$
\liminf _{n \rightarrow \infty} \operatorname{Rot}_{(2.2)}\left(t_{n} ; u_{n}\right) \geq \frac{\bar{\theta}(\tau)-\alpha}{\pi}=\frac{\bar{\theta}(\tau)-\bar{\theta}(0)}{\pi} .
$$

Finally, recalling (3.13) and the definition of $\operatorname{Rot}_{\varphi-\delta, \psi-\delta}(\tau ; w)$, we have

$$
\operatorname{Rot}_{\varphi-\delta, \psi-\delta}(\tau ; w) \leq \operatorname{Rot}_{\varphi, \psi}(\tau ; w)-\varepsilon
$$

This, clearly, contradicts (3.9).

We have thus proved the first claim in Lemma 3.1. The proof of the other three parts of the statement follows precisely the same steps (even with some simplification for the case of rotations near zero) and therefore it is omitted. 
In order to apply Theorem 2.1, we will now state four propositions (analogous to the ones described in [16]) which provide the needed estimates on the rotation number of a solution to the initial value problem

$$
\begin{gathered}
u^{\prime \prime}(t)+f(t, u(t))=0, \\
u(0)=0, \quad u^{\prime}(0)=z_{0},
\end{gathered}
$$

with $z_{0} \in \mathbb{R}$ suitably chosen.

The first proposition provides a bound from below on the rotation number for all the solutions of (3.20) having $z_{0}$ "large enough."

Proposition 3.2. Suppose that $f$ satisfies the hypothesis $\left(H^{ \pm}\right)$and the Carathéodory conditions. Moreover, assume that there exists $j \in \mathbb{N}, v \in\{<,>\}$ such that $\lambda_{j}^{v}\left(a_{\infty}^{+}, a_{\infty}^{-}\right)<1$. Then, there exists $\mathscr{R}>0$ such that for every $z_{0} \in \mathbb{R}$ with $z_{0} v 0,\left|z_{0}\right|>\mathscr{R}$, every (possible) solution $u$ of (3.20) is such that $\operatorname{Rot}_{(2.2)}(T ; u)>j$.

Proof. We take $z_{0} \in \mathbb{R}, z_{0} \nu 0$, and a solution $u$ of (3.20). Moreover, we define $v:=$ $\left(0, z_{0} /\left|z_{0}\right|\right)$. Since $\lambda_{j}^{v}\left(a_{\infty}^{+}, a_{\infty}^{-}\right)<1$, Lemma 2.6 implies that $\operatorname{Rot}_{a_{\infty}^{+}, a_{\infty}^{-}}(T ; v)>j$. Thus, it is possible to choose a positive constant $\varepsilon$ satisfying

$$
\operatorname{Rot}_{a_{\infty}^{+}, a_{\infty}^{-}}(T ; v)-\varepsilon>j .
$$

We note that from hypothesis $\left(\mathrm{H}^{ \pm}\right)$the continuous functions $a_{\infty}^{+}$and $a_{\infty}^{-}$satisfy, respectively, the assumptions (3.1) in Lemma 3.1. Hence, by applying such a lemma, we deduce the existence of a constant $R=R(\varepsilon)>0$ such that if $\left|\left(u(t), u^{\prime}(t)\right)\right| \geq R$ for all $t \in[0, T]$, then

$$
\operatorname{Rot}_{(2.2)}(t ; u) \geq \operatorname{Rot}_{a_{\infty}^{+}, a_{\infty}^{-}}(t ; v)-\varepsilon, \quad \forall t \in[0, T],
$$

and, in particular, $\operatorname{Rot}_{(2.2)}(T ; u)>j$.

In order to determine a lower bound on $\left|z_{0}\right|$ which leads to $\left|\left(u(t), u^{\prime}(t)\right)\right| \geq R$ for every $t \in[0, T]$, we will apply Lemma 2.7 , since its hypotheses are verified. Indeed, by combining the assumption $\left(\mathrm{H}^{ \pm}\right)$with the Carathéodory conditions, for $\sigma>0$, we can find a function $C=C\left(a_{\infty}^{ \pm}, b_{\infty}^{ \pm}, \sigma\right) \in C\left([0, T], \mathbb{R}_{+}\right)$and a function $\ell=\ell_{\sigma} \in \mathrm{L}^{1}\left([0, T], \mathbb{R}_{+}\right)$such that

$$
|f(t, x)| \leq C(t)|x|+\ell(t), \quad \forall x \in \mathbb{R} \text {, for a.e. } t \in[0, T] .
$$

Thus, setting $R_{1}=R$ in Lemma 2.7, we infer that there exists $\mathscr{R}>R$ such that if $\left|z_{0}\right|>\mathscr{R}$, then $\left|\left(u(t), u^{\prime}(t)\right)\right|>R$ for every $t \in[0, T]$. This completes the proof.

The following proposition guarantees a bound from above on the rotation number of all the (possible) solutions to (3.20) for which $z_{0}$ is taken sufficiently large. Since the corresponding proof follows the same steps of Proposition 3.2, we will omit the details.

Proposition 3.3. Suppose that $f$ satisfies the hypothesis $\left(H^{ \pm}\right)$and the Carathéodory conditions. Moreover, assume that there exists $j \in \mathbb{N}$ and $\nu \in\{<,>\}$ such that $\lambda_{j}^{\nu}\left(b_{\infty}^{+}, b_{\infty}^{-}\right)>1$. Then, there exists $\mathscr{R}^{*}>0$ such that for every $z_{0} \in \mathbb{R}$ with $z_{0} v 0$ and $\left|z_{0}\right|>\mathscr{R}^{*}$, every (possible) solution $u$ of $(3.20)$ is such that $\operatorname{Rot}_{(2.2)}(T ; u)<j$. 
Finally, the last two propositions provide lower and upper estimates, respectively, for the rotation number of all the solutions of (3.20) characterized by a sufficiently small $z_{0}$. Both their proofs are similar to the one of Proposition 3.2. The only difference consists in the fact that Lemma 2.8 (instead of Lemma 2.7) is used in order to achieve the result. For this reason, we will prove only the following.

Proposition 3.4. Suppose that $f$ satisfies the hypothesis $\left(K^{ \pm}\right)$and the Carathéodory conditions. Moreover, assume that there exists $i \in \mathbb{N}$ and $\nu \in\{<,>\}$ such that $\lambda_{i}^{v}\left(a_{0}^{+}, a_{0}^{-}\right)<1$. Then, there exists $\delta>0$ such that for every $z_{0} \in \mathbb{R}$ with $z_{0} \nu 0$ and $\left|z_{0}\right|<\delta$, every (possible) solution $u$ of (3.20) is such that $\operatorname{Rot}_{(2.2)}(T ; u)>i$.

Proof. We take $z_{0} \in \mathbb{R}$ with $z_{0} \nu 0$ and a solution $u$ of (3.20). Since $\lambda_{i}^{\nu}\left(a_{0}^{+}, a_{0}^{-}\right)<1$, Lemma 2.6 implies that $\operatorname{Rot}_{a_{0}^{+}, a_{0}^{-}}(T ; v)>i$, where $v:=\left(0, z_{0} /\left|z_{0}\right|\right)$. Thus, it is possible to choose a positive constant $\varepsilon$ satisfying

$$
\operatorname{Rot}_{a_{0}^{+}, a_{0}^{-}}(T ; v)-\varepsilon>i
$$

Hypothesis $\left(\mathrm{K}^{ \pm}\right)$ensures that conditions in (3.5) are satisfied by $a_{0}^{+}, a_{0}^{-}$, respectively. Hence, by Lemma 3.1, we deduce the existence of a constant $r=r(\varepsilon)>0$ such that if $0<\left|\left(u(t), u^{\prime}(t)\right)\right| \leq r$ for all $t \in[0, T]$, then

$$
\operatorname{Rot}_{(2.2)}(T ; u) \geq \operatorname{Rot}_{a_{0}^{+}, a_{0}^{-}}(T ; v)-\varepsilon>i
$$

We observe that the hypotheses of Lemma 2.8 are verified, since from the assumption $\left(\mathrm{K}^{ \pm}\right)$, fixed $\eta>0$, we can find a constant $\sigma=\sigma(\eta)>0$ and a continuous function $D=$ $D\left(a_{0}^{ \pm}, b_{0}^{ \pm}, \eta\right):[0, T] \rightarrow \mathbb{R}_{+}$such that

$$
|f(t, x)| \leq D(t)|x|, \quad \forall x \in \mathbb{R}:|x|<\sigma \text {, for a.e. } t \in[0, T] .
$$

Lemma 2.8 guarantees that whenever $z_{0}$ is different from zero, then $\left|\left(u(t), u^{\prime}(t)\right)\right|>0$ for every $t \in[0, T]$.

In order to determine an upper bound on $\left|z_{0}\right|$ which leads to $\left|\left(u(t), u^{\prime}(t)\right)\right| \leq r$ for every $t \in[0, T]$, we use again Lemma 2.8. In particular, setting $\rho_{1}=r$, we conclude that there exists $\delta \in(0, r)$ such that if $\left|z_{0}\right| \leq \delta$, then $\left|\left(u(t), u^{\prime}(t)\right)\right| \leq r$ for every $t \in[0, T]$. This completes the proof.

Proposition 3.5. Suppose that $f$ satisfies the hypothesis $\left(K^{ \pm}\right)$and the Caratheodory conditions. Moreover, assume that there exists $i \in \mathbb{N}$ and $\nu \in\{<,>\}$ such that $\lambda_{i}^{v}\left(b_{0}^{+}, b_{0}^{-}\right)>1$. Then, there exists $\delta^{*}>0$ such that for every $z_{0} \in \mathbb{R}$ with $z_{0} \nu 0$ and $\left|z_{0}\right|<\delta^{*}$, every (possible) solution $u$ of (3.20) is such that $\operatorname{Rot}_{(2.2)}(T ; u)<i$.

Remark 3.6. We note that Proposition 3.3 holds independently on the sign of $a_{\infty}^{ \pm}$, provided we assume the less restrictive condition $\left(\left(b_{\infty}^{+}\right)^{+},\left(b_{\infty}^{-}\right)^{+}\right) \succ(0,0)$ on $(0, T)$.

A symmetric observation could be written for Proposition 3.5. 
Now we prove our main result, Theorem 1.2. Theorem 1.2 can be easily proved combining the previous propositions with Theorem 2.1.

Proof. We take $\nu \in\{<,>\}$ and $h \in \mathbb{N}$ with $m \leq h \leq n$. First of all, assume that $\lambda_{n}^{\nu}\left(a_{0}^{+}, a_{0}^{-}\right)<$ $1<\lambda_{m}^{v}\left(b_{\infty}^{+}, b_{\infty}^{-}\right)$. In particular, by Proposition 3.3, we can find a positive constant $\mathscr{R}^{*}$ such that for every $y_{0} \in \mathbb{R}$ with $y_{0} \nu 0$ and $\left|y_{0}\right|>\mathscr{R}^{*}$, every (possible) solution $u$ of

$$
\begin{gathered}
u^{\prime \prime}(t)+f(t, u(t))=0, \\
u(0)=0, \quad u^{\prime}(0)=y_{0}
\end{gathered}
$$

is such that $\operatorname{Rot}_{(2.2)}(T ; u)<m \leq h$. Moreover, from Proposition 3.4 it follows that there exists $\delta>0$ such that for every $y_{0} \in \mathbb{R}$ with $y_{0} \nu 0$ and $\left|y_{0}\right|<\delta$, every (possible) solution $u$ of (3.27) is such that $\operatorname{Rot}_{(2.2)}(T ; u)>n \geq h$. It is not restrictive to suppose that $\delta<\mathscr{R}^{*}$. Taking into account Lemmas 2.7 and 2.8 and fixing $r \in(0, \delta)$ and $R \in\left(\mathscr{R}^{*},+\infty\right)$, we observe that all the hypotheses of Theorem 2.1 are satisfied. Thus, Theorem 1.2 is proved in this first case.

If $\lambda_{n}^{v}\left(a_{\infty}^{+}, a_{\infty}^{-}\right)<1<\lambda_{m}^{v}\left(b_{0}^{+}, b_{0}^{-}\right)$, we use Propositions 3.2 and 3.5 in order to obtain the estimates on the rotation number. Hence, following exactly the previous steps, from an application of Theorem 2.1 we easily achieve the thesis.

Now, we provide two comparison remarks with the multiplicity results obtained in an asymmetric, asymptotically linear setting by Dinca and Sanchez in [20] and by Sadyrbaev in [48], respectively.

Remark 3.7. The paper [20] deals with the existence and multiplicity of solutions for an asymmetric problem with constant weights of the form (1.1) satisfying the following asymptotically linear assumptions.

There exist $\varepsilon_{0}>0, R>0$, and positive constants $a_{0}^{ \pm}, b_{0}^{ \pm}, a_{\infty}^{ \pm}, b_{\infty}^{ \pm}$with $0<a_{0}^{ \pm} \leq b_{0}^{ \pm}, 0<$ $a_{\infty}^{ \pm} \leq b_{\infty}^{ \pm}$, such that

$$
\begin{array}{ll}
a_{0}^{+} \leq \frac{f(t, u)}{u} \leq b_{0}^{+} & \forall(t, u) \in \mathbb{R} \times] 0, \varepsilon_{0}[, \\
a_{0}^{-} \leq \frac{f(t, u)}{u} \leq b_{0}^{-} & \forall(t, u) \in \mathbb{R} \times]-\varepsilon_{0}, 0[, \\
a_{\infty}^{+} \leq \frac{f(t, u)}{u} \leq b_{\infty}^{+} & \forall(t, u) \in \mathbb{R} \times] R,+\infty[, \\
a_{\infty}^{-} \leq \frac{f(t, u)}{u} \leq b_{\infty}^{-} & \forall(t, u) \in \mathbb{R} \times]-\infty, R[.
\end{array}
$$

A Lipschitz condition on $f$ is required as well. In order to achieve the multiplicity result, in [20] a shooting argument is developed based on the notion of "variation index" (see also [31]) and the implicit function theorem is applied. We point out that [20, Theorem 3 (a) and (c)] follow from Theorem 1.2, while, in some situations, [20, Theorem 3(b) and (d)] might provide an extra solution. 
974 Multiplicity results for asymmetric BVPs with weights

The gap condition (analogous to our assumptions (1.6) or (1.7)) between the behavior of the nonlinearity in zero and near infinity is written in terms of the number of zeros $k_{\nu}(\lambda, \mu)$ in $(0, \pi)$ of the solution of

$$
u^{\prime \prime}+\lambda u^{+}-\mu u^{-}=0, \quad u(0)=0, \quad\left|u^{\prime}(0)\right|=1, \quad u^{\prime}(0) v 0,
$$

where $\lambda, \mu$ are positive constants and $\nu \in\{<,>\}$.

Statements (a) and (c) in [20, Theorem 3] ensure the existence of $k_{\nu}\left(a_{0}^{+}, a_{0}^{-}\right)-k_{\nu}\left(b_{\infty}^{+}\right.$, $\left.b_{\infty}^{-}\right)$solutions, under the further hypotheses

$$
k_{\nu}\left(a_{0}^{+}, a_{0}^{-}\right)>k_{\nu}\left(b_{\infty}^{+}, b_{\infty}^{-}\right)
$$

and

$$
\operatorname{Rot}_{b_{\infty}^{+}, b_{\infty}^{-}}\left(\pi ; v_{\nu}\right) \notin \mathbb{N}, \quad v_{>}:=(0,1), v_{<}:=(0,-1) .
$$

We now compare the assumption (1.6) with the assumptions of [20, Theorem 3(a) and (c)]. To this aim, we rewrite them in terms of the rotation numbers $\operatorname{Rot}_{b_{\infty}^{+}, b_{\infty}^{-}}\left(\pi ; v_{\nu}\right)$ and $\operatorname{Rot}_{a_{0}^{+}, a_{0}^{-}}\left(\pi ; v_{\nu}\right)$.

By Lemma 2.6 our hypothesis (1.6) is equivalent to the following:

$$
\exists n, m \in \mathbb{N}, m \leq n: \operatorname{Rot}_{a_{0}^{+}, a_{0}^{-}}\left(\pi ; v_{\nu}\right)>n \geq m>\operatorname{Rot}_{b_{\infty}^{+}, b_{\infty}^{-}}\left(\pi ; v_{\nu}\right) .
$$

We recall that Theorem 1.2 guarantees the existence of $n-m+1$ solutions whenever (3.32) holds.

On the other hand, the number of zeros $k_{v}$ can be equivalently defined by the following expression:

$$
\begin{aligned}
k_{\nu}\left(a_{0}^{+}, a_{0}^{-}\right) & =\max \left\{m \in \mathbb{N}: m<\operatorname{Rot}_{a_{0}^{+}, a_{0}^{-}}\left(\pi ; v_{\nu}\right)\right\}, \\
k_{\nu}\left(b_{\infty}^{+}, b_{\infty}^{-}\right) & =\max \left\{m \in \mathbb{N}: m<\operatorname{Rot}_{b_{\infty}^{+}, b_{\infty}^{-}}\left(\pi ; v_{\nu}\right)\right\} .
\end{aligned}
$$

Thus, inequality (3.30) implies that

$$
\operatorname{Rot}_{a_{0}^{+}, a_{0}^{-}}\left(\pi ; v_{v}\right)>k_{\nu}\left(a_{0}^{+}, a_{0}^{-}\right)>k_{\nu}\left(b_{\infty}^{+}, b_{\infty}^{-}\right),
$$

which, combined with (3.31), leads to

$$
\operatorname{Rot}_{a_{0}^{+}, a_{0}^{-}}\left(\pi ; v_{\nu}\right)>k_{\nu}\left(a_{0}^{+}, a_{0}^{-}\right) \geq k_{\nu}\left(b_{\infty}^{+}, b_{\infty}^{-}\right)+1>\operatorname{Rot}_{b_{\infty}^{+}, b_{\infty}^{-}}\left(\pi ; v_{\nu}\right) .
$$

Such inequalities guarantee that conditions (3.32) and, equivalently, (1.6) in Theorem 1.2 are satisfied. In particular, our Theorem 1.2 provides the existence of $k_{\nu}\left(a_{0}^{+}, a_{0}^{-}\right)-k_{\nu}\left(b_{\infty}^{+}\right.$, $\left.b_{\infty}^{-}\right)$solutions to problem (1.1). Hence, we can conclude that [20, Theorem 3(a) and (c)] follow from our main result.

On the other hand, [20, Theorem 3(b) and (d)], dealing with multiplicity of solutions to problem (1.1) whenever a gap condition involving the constants $a_{\infty}^{ \pm}$and $b_{0}^{ \pm}$holds, are more general than the ones we have analyzed above, since a condition symmetric to (3.31) is not assumed. 
Remark 3.8. In [48], Sadyrbaev has given a multiplicity result for a scalar equation of the form

$$
u^{\prime \prime}+f(t, u)=g\left(t, u, u^{\prime}\right)
$$

where $g$ is sublinear and $f$ is asymptotically linear at infinity. More precisely, it is supposed that $\lim _{x \rightarrow+\infty}(f(t, x) / x)=c_{\infty}^{+}(t)$ and $\lim _{x \rightarrow-\infty}(f(t, x) / x)=c_{\infty}^{-}(t)$ uniformly in $t$.

The argument in [48] is developed through the study of some variation equation (cf. $[48,(1.5)])$ associated to (3.36) and assumptions are given relatively to the number of zeros of the solutions of this auxiliary equation and of the two-weighted, half-linear equation

$$
u^{\prime \prime}(t)+c_{\infty}^{+}(t) u^{+}(t)-c_{\infty}^{-}(t) u^{-}(t)=0, \quad\left(u(0), u^{\prime}(0)\right)=v_{\gamma}
$$

For this approach, it is required that the functions $f$ and $g$ be of class $C^{1}$. No explicit sign condition is assumed on the continuous functions $c_{\infty}^{ \pm}$.

It is possible to compare our main result with the one of Sadyrbaev provided that we assume $g \equiv 0,\left(c_{\infty}^{+}\right)^{+} \cdot\left(c_{\infty}^{-}\right)^{+} \not \equiv 0$, and $\lim _{x \rightarrow 0}(f(t, x) / x)=c_{0}(t)$ uniformly in $t$, where $c_{0}^{+} \not \equiv 0$. In this particular situation, the variation equation studied in [48] becomes

$$
u^{\prime \prime}(t)+c_{0}(t) u(t)=0, \quad u(0)=0, \quad u^{\prime}(0)=1
$$

Arguing as in Remark 3.7, one can transform the hypotheses in [48] involving the number of zeros of the solutions to the Cauchy problems (3.37) and (3.38) into hypotheses involving the weighted eigenvalues. More precisely, the assumptions of [48, Corollary 4.1] can be rewritten as follows: for each $\nu \in\{<\rangle$,$\} there exist h_{\nu}, k \in \mathbb{N}$ (which represent the number of zeros of the solutions to (3.37) and (3.38), resp.) such that

$$
\lambda_{h_{\nu}}^{v}\left(c_{\infty}^{+}, c_{\infty}^{-}\right)<1<\lambda_{h_{\nu}+1}^{\nu}\left(c_{\infty}^{+}, c_{\infty}^{-}\right), \quad \lambda_{k}^{v}\left(c_{0}, c_{0}\right)<1<\lambda_{k+1}^{v}\left(c_{0}, c_{0}\right)
$$

In this setting, [48, Corollary 4.1] provides the existence of at least $\left|h_{>}-k\right|+\left|k-h_{<}\right|$ nontrivial solutions to problem (1.1). It is easy to show that such a corollary follows from Theorem 1.2.

Remark 3.9. Rynne in [47] has studied multiplicity of solutions for asymmetric problems involving superlinear nonlinearities. Multiplicity is achieved by using an alternative approach. Instead of an eigenvalue problem of the form (1.4), [47] considers the halfeigenvalues problem

$$
\begin{gathered}
u^{\prime \prime}+\mu u(t)+\varphi(t) u^{+}(t)-\psi(t) u^{-}(t)=0, \\
u(0)=0=u(T), \quad u^{\prime}(0) \nu 0,
\end{gathered}
$$

where $\varphi, \psi \in \mathrm{L}^{\infty}([0, T], \mathbb{R})$ and $\nu \in\{<,>\}$. The corresponding eigenvalue theory is developed, among others, in [7, Theorem 2], where $\varphi, \psi$ are continuous functions on $[0, T]$, 
and, more recently, in $\left[45\right.$, Theorem 5.1] for $\varphi, \psi \in \mathrm{L}^{\infty}([0, T], \mathbb{R})$. In particular, [45, Theorem 5.1] is the analog (in this alternative setting) of Theorem 1.1 and provides a monotone increasing sequence of half-eigenvalues

$$
\mu_{1}^{\nu}(\varphi, \psi)<\mu_{2}^{\nu}(\varphi, \psi)<\cdots<\mu_{j}^{\nu}(\varphi, \psi)<\cdots
$$

for problem (3.40). The half-eigenfunction corresponding to $\mu_{j}^{\nu}(\varphi, \psi)$ has exactly $j-1$ zeros on $(0, T)$.

By taking into account the nodal properties of the half-eigenfunctions, the Sturm comparison Lemma 2.3, and the relations in Lemma 2.6, it is immediate to deduce the validity of the following equivalences between the half-eigenvalues $\mu_{j}^{\nu}(\varphi, \psi)$ and $\lambda_{j}^{\nu}(\varphi, \psi)$ :

$$
\begin{aligned}
\mu_{j}^{\nu}(\varphi, \psi)<0 & \Longleftrightarrow \operatorname{Rot}_{\varphi, \psi}\left(T ; v_{\nu}\right)>\operatorname{Rot}_{\mu_{j}^{v}(\varphi, \psi)+\varphi, \mu_{j}^{v}(\varphi, \psi)+\psi}\left(T ; v_{\nu}\right)=j \\
& \Longleftrightarrow \lambda_{j}^{\nu}(\varphi, \psi)<1 .
\end{aligned}
$$

Analogous relations are satisfied whenever we reverse the inequalities.

According to (3.42), we infer that Theorem 1.2 holds true even if we replace assumptions (1.6) and (1.7) with the equivalent conditions

$$
\mu_{n}^{v}\left(a_{0}^{+}, a_{0}^{-}\right)<0<\mu_{m}^{v}\left(b_{\infty}^{+}, b_{\infty}^{-}\right)
$$

and

$$
\mu_{n}^{\nu}\left(a_{\infty}^{+}, a_{\infty}^{-}\right)<0<\mu_{m}^{\nu}\left(b_{0}^{+}, b_{0}^{-}\right)
$$

respectively.

Rynne in [47] proves the existence of solutions with specified nodal properties to some asymmetric problem by studying the sign of the eigenvalues $\mu_{j}^{\nu}(\varphi, \psi)$ associated to the two-weighted, half-linear problem (3.40). The result in [47] is not directly comparable with ours, since in [47] a superlinear assumption near $+\infty$ is considered.

We point out that sign conditions like $\mu_{j}^{v}(\varphi, \psi)<0$ implicitly contain some hypothesis on the sign of the weights $\varphi, \psi$.

\section{Acknowledgments}

The author wishes to thank Professor Anna Capietto and Professor Fabio Zanolin for their useful suggestions. This work was performed in the frame of the project GNAMPA 2003 "Ordinary Differential Equations: Theory and Applications" and of the project COFIN 2001 "Variational and Topological Methods for the Study of Nonlinear Phenomena."

\section{References}

[1] M. Alif, Sur le spectre de Fučík du p-Laplacien avec des poids indéfinis [On the spectrum of Fučík of the p-Laplacian with indefinite weights], C. R. Math. Acad. Sci. Paris 334 (2002), no. 12, 1061-1066 (French).

[2] M. Alif and J. P. Gossez, On the Fučik spectrum with indefinite weights, Differential Integral Equations 14 (2001), no. 12, 1511-1530. 
[3] H. Amann, Fixed point equations and nonlinear eigenvalue problems in ordered Banach spaces, SIAM Rev. 18 (1976), no. 4, 620-709.

[4] H. Amann and E. Zehnder, Nontrivial solutions for a class of nonresonance problems and applications to nonlinear differential equations, Ann. Scuola Norm. Sup. Pisa Cl. Sci. (4) 7 (1980), no. 4, 539-603.

[5] M. Arias, J. Campos, M. Cuesta, and J. P. Gossez, Asymmetric elliptic problems with indefinite weights, Ann. Inst. H. Poincaré Anal. Non Linéaire 19 (2002), no. 5, 581-616.

[6] T. Bartsch and Z. Q. Wang, On the existence of sign changing solutions for semilinear Dirichlet problems, Topol. Methods Nonlinear Anal. 7 (1996), no. 1, 115-131.

[7] H. Berestycki, On some nonlinear Sturm-Liouville problems, J. Differential Equations 26 (1977), no. 3, 375-390.

[8] A. Cañada, P. Drábek, and J. L. Gámez, Existence of positive solutions for some problems with nonlinear diffusion, Trans. Amer. Math. Soc. 349 (1997), no. 10, 4231-4249.

[9] A. Capietto and F. Dalbono, Multiplicity results for systems of asymptotically linear second order equations, Adv. Nonlinear Stud. 2 (2002), no. 4, 325-356.

[10] A. Capozzi, D. Lupo, and S. Solimini, On the existence of a nontrivial solution to nonlinear problems at resonance, Nonlinear Anal. 13 (1989), no. 2, 151-163.

[11] A. Castro and A. C. Lazer, Critical point theory and the number of solutions of a nonlinear Dirichlet problem, Ann. Mat. Pura Appl. (4) 120 (1979), 113-137.

[12] E. A. Coddington and N. Levinson, Theory of Ordinary Differential Equations, McGraw-Hill Book Company, New York, 1955.

[13] F. J. S. A. Corrêa, On pairs of positive solutions for a class of sub-superlinear elliptic problems, Differential Integral Equations 5 (1992), no. 2, 387-392.

[14] D. G. Costa, D. G. de Figueiredo, and P. N. Srikanth, The exact number of solutions for a class of ordinary differential equations through Morse index computation, J. Differential Equations 96 (1992), no. 1, 185-199.

[15] F. Dalbono, Multiplicity results for superlinear and asymptotically linear boundary value problems, Ph.D. thesis, Università di Genova, Genova, 2002.

[16] F. Dalbono and F. Zanolin, Multiplicity results for asymptotically linear equations, using the rotation number approach, preprint, 2002.

[17] E. N. Dancer, On the ranges of certain weakly nonlinear elliptic partial differential equations, J. Math. Pures Appl. (9) 57 (1978), no. 4, 351-366.

[18] D. G. de Figueiredo, Positive solutions of semilinear elliptic problems, Differential Equations (São Paulo, 1981), Lecture Notes in Math., vol. 957, Springer, Berlin, 1982, pp. 34-87.

[19] D. G. de Figueiredo and O. H. Miyagaki, Semilinear elliptic equations with the primitive of the nonlinearity away from the spectrum, Nonlinear Anal. 17 (1991), no. 12, 1201-1219.

[20] G. Dinca and L. Sanchez, Multiple solutions of boundary value problems: an elementary approach via the shooting method, NoDEA Nonlinear Differential Equations Appl. 1 (1994), no. 2, 163-178.

[21] Y. Dong, On the solvability of asymptotically positively homogeneous equations with SturmLiouville boundary value conditions, Nonlinear Anal. 42 (2000), no. 8, 1351-1363.

[22] M. J. Esteban, Multiple solutions of semilinear elliptic problems in a ball, J. Differential Equations 57 (1985), no. 1, 112-137.

[23] D. Fortunato, Morse theory and nonlinear elliptic problems, Progress in Elliptic and Parabolic Partial Differential Equations (Capri, 1994), Pitman Res. Notes Math. Ser., vol. 350, Longman, Harlow, 1996, pp. 163-172.

[24] S. Fučík, Boundary value problems with jumping nonlinearities, Časopis Pěst. Mat. 101 (1976), no. $1,69-87$.

[25] M. García-Huidobro, R. Manásevich, and F. Zanolin, A Fredholm-like result for strongly nonlinear second order ODEs, J. Differential Equations 114 (1994), no. 1, 132-167. 
[26] M. García-Huidobro and P. Ubilla, Multiplicity of solutions for a class of nonlinear second-order equations, Nonlinear Anal. 28 (1997), no. 9, 1509-1520.

[27] J. K. Hale, Ordinary Differential Equations, Robert E. Krieger Publishing, New York, 1980.

[28] G. Harris and B. Zinner, Some remarks concerning exact solution numbers for a class of nonlinear boundary value problems, J. Math. Anal. Appl. 182 (1994), no. 3, 571-588.

[29] N. Hirano, Multiple nontrivial solutions of semilinear elliptic equations, Proc. Amer. Math. Soc. 103 (1988), no. 2, 468-472.

[30] Y. X. Huang, Existence of positive solutions for a class of the p-Laplace equations, J. Austral. Math. Soc. Ser. B 36 (1994), no. 2, 249-264.

[31] H. G. Kaper and M. K. Kwong, On two conjectures concerning the multiplicity of solutions of a Dirichlet problem, SIAM J. Math. Anal. 23 (1992), no. 3, 571-578.

[32] Y. A. Klokov and F. Z. Sadyrbaev, On the number of solutions of second-order boundary value problems with nonlinear asymptotics, Differ. Uravn. 34 (1998), no. 4, 471-479 (Russian).

[33] M. A. Krasnosel'skiy, A. I. Perov, A. I. Povolotskiy, and P. P. Zabreiko, Plane Vector Fields, Academic Press, New York, 1966.

[34] N. Lakoš, Positive solutions of systems of nonlinear eigenvalue problems, Nonlinear Anal. 16 (1991), no. 1, 89-98.

[35] V. Lakshmikantham and S. Leela, Differential and Integral Inequalities: Theory and Applications. Vol. I: Ordinary Differential Equations, Academic Press, New York, 1969.

[36] A. C. Lazer and P. J. McKenna, On a conjecture related to the number of solutions of a nonlinear Dirichlet problem, Proc. Roy. Soc. Edinburgh Sect. A 95 (1983), no. 3-4, 275-283.

[37] G. Li and H. S. Zhou, Multiple solutions to p-Laplacian problems with asymptotic nonlinearity as $u^{p-1}$ at infinity, J. London Math. Soc. (2) 65 (2002), no. 1, 123-138.

[38] S. Li and J. Su, Existence of multiple solutions of a two-point boundary value problem at resonance, Topol. Methods Nonlinear Anal. 10 (1997), no. 1, 123-135.

[39] S. Li and M. Willem, Multiple solutions for asymptotically linear boundary value problems in which the nonlinearity crosses at least one eigenvalue, NoDEA Nonlinear Differential Equations Appl. 5 (1998), no. 4, 479-490.

[40] S. J. Li and J. Q. Liu, Nontrivial critical points for asymptotically quadratic function, J. Math. Anal. Appl. 165 (1992), no. 2, 333-345.

[41] A. Marino, A. M. Micheletti, and A. Pistoia, A nonsymmetric asymptotically linear elliptic problem, Topol. Methods Nonlinear Anal. 4 (1994), no. 2, 289-339.

[42] J. Mawhin, C. Rebelo, and F. Zanolin, Continuation theorems for Ambrosetti-Prodi type periodic problems, Commun. Contemp. Math. 2 (2000), no. 1, 87-126.

[43] F. I. Njoku and F. Zanolin, Positive solutions for two-point BVPs: existence and multiplicity results, Nonlinear Anal. 13 (1989), no. 11, 1329-1338.

[44] W. Reichel and W. Walter, Sturm-Liouville type problems for the p-Laplacian under asymptotic non-resonance conditions, J. Differential Equations 156 (1999), no. 1, 50-70.

[45] B. P. Rynne, The Fučík spectrum of general Sturm-Liouville problems, J. Differential Equations 161 (2000), no. 1, 87-109.

[46] Non-resonance conditions for semilinear Sturm-Liouville problems with jumping nonlinearities, J. Differential Equations 170 (2001), no. 1, 215-227.

[47] - Second-order Sturm-Liouville problems with asymmetric, superlinear nonlinearities, Nonlinear Anal. 54 (2003), no. 5, 939-947.

[48] F. Sadyrbaev, Multiplicity of solutions for two-point boundary value problems with asymptotically asymmetric nonlinearities, Nonlinear Anal. 27 (1996), no. 9, 999-1012.

[49] P. Ubilla, Multiplicity results for the 1-dimensional generalized p-Laplacian, J. Math. Anal. Appl. 190 (1995), no. 2, 611-623.

[50] C. Zanini, Rotation numbers, eigenvalues, and the Poincaré-Birkhoff theorem, J. Math. Anal. Appl. 279 (2003), no. 1, 290-307. 
[51] M. Zhang, Certain classes of potentials for p-Laplacian to be non-degenerate, preprint, 2004.

[52] , Nonuniform nonresonance of semilinear differential equations, J. Differential Equations 166 (2000), no. 1, 33-50.

[53] The rotation number approach to the periodic Fučik spectrum, J. Differential Equations 185 (2002), no. 1, 74-96.

[54] W. Zou, Multiple solutions results for two-point boundary value problem with resonance, Discrete Contin. Dyn. Syst. 4 (1998), no. 3, 485-496.

Francesca Dalbono: Dipartimento di Matematica, Facoltà di Scienze Matematiche Fisiche e Naturali, Università di Torino, Via Carlo Alberto, 1010123 Torino, Italy

E-mail address: dalbono@dm.unito.it 


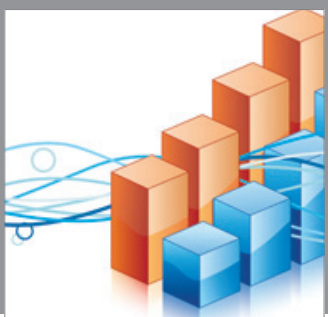

Advances in

Operations Research

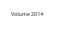

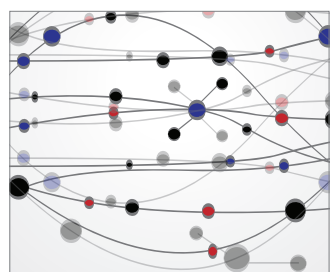

\section{The Scientific} World Journal
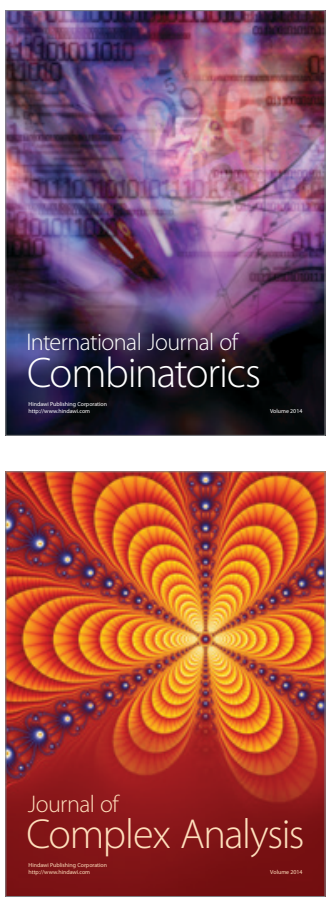

International Journal of

Mathematics and

Mathematical

Sciences
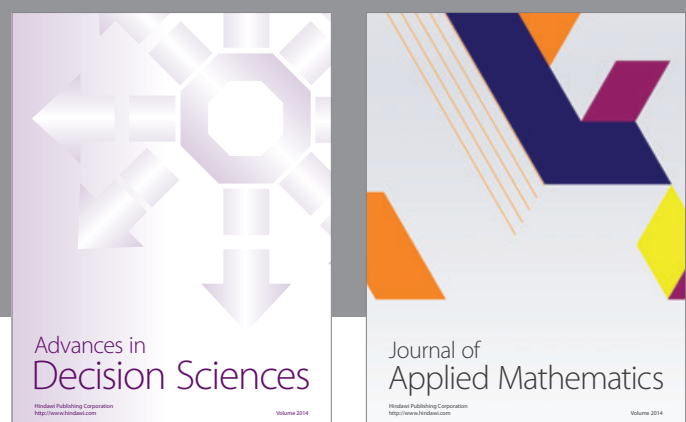

Journal of

Applied Mathematics
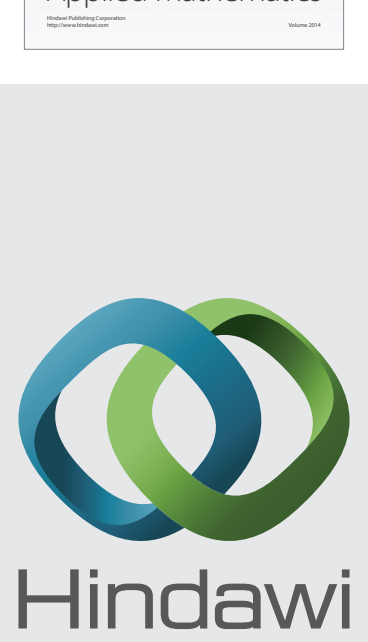

Submit your manuscripts at http://www.hindawi.com
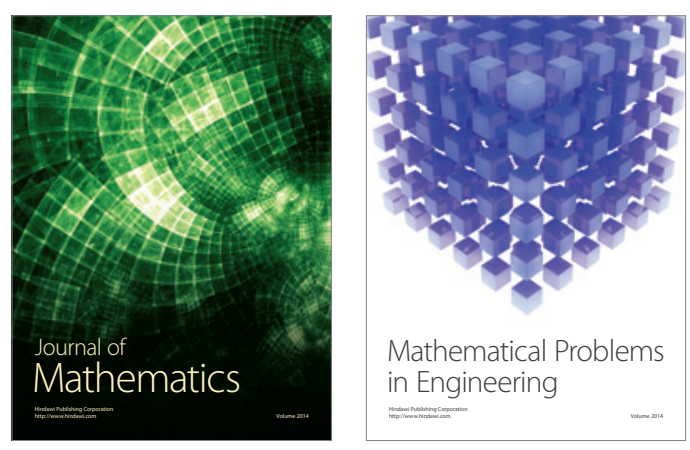

Mathematical Problems in Engineering
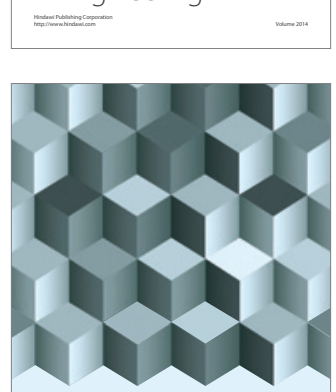

Journal of

Function Spaces
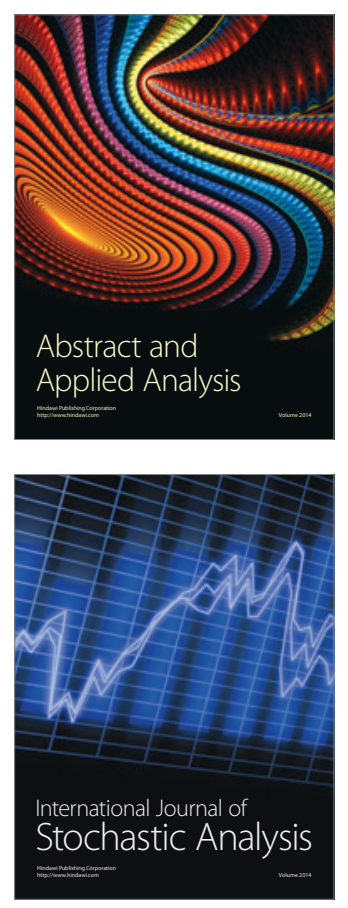

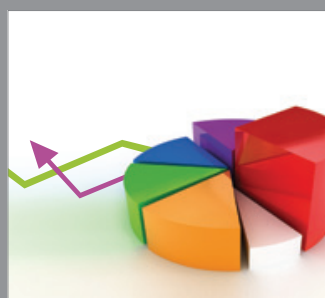

ournal of

Probability and Statistics

Promensencen
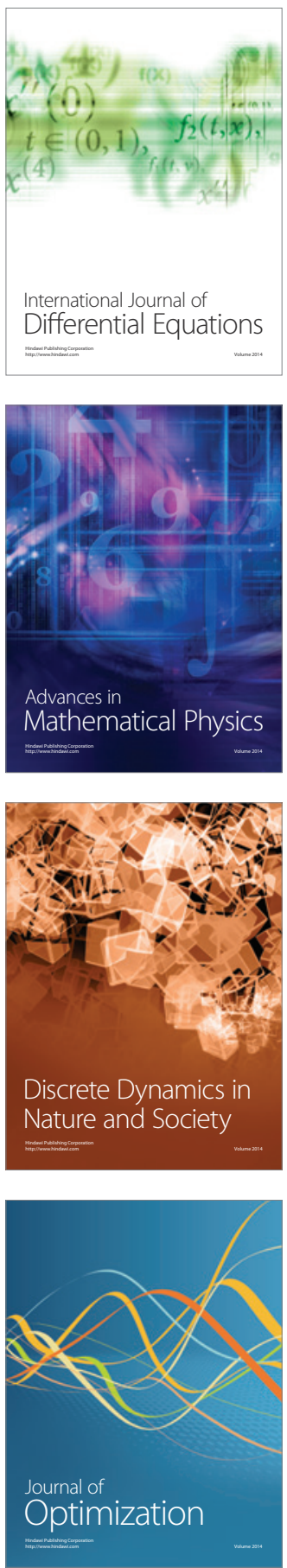